\title{
Pharmacokinetics of the Perioperative Use of Cancer Chemotherapy in Peritoneal Surface Malignancy Patients
}

\author{
K. Van der Speeten, ${ }^{1,2,3}$ K. Govaerts, ${ }^{1}$ O. A. Stuart, ${ }^{3}$ and P. H. Sugarbaker ${ }^{3}$ \\ ${ }^{1}$ Department of Surgical Oncology, Oost-Limburg Hospital, Schiepse Bos 6, 3600 Genk, Belgium \\ ${ }^{2}$ Department of Biochemistry, Faculty of Medicine, University Hasselt, 3590 Diepenbeek, Belgium \\ ${ }^{3}$ Washington Cancer Institute, Washington Hospital Center, Washington, DC 20010, USA
}

Correspondence should be addressed to K. Van der Speeten, kurt.vanderspeeten@zol.be

Received 10 March 2012; Accepted 23 April 2012

Academic Editor: Yan Li

Copyright (c) 2012 K. Van der Speeten et al. This is an open access article distributed under the Creative Commons Attribution License, which permits unrestricted use, distribution, and reproduction in any medium, provided the original work is properly cited.

Background. The peritoneal surface is an acknowledged locoregional failure site of abdominal malignancies. Previous treatment attempts with medical therapy alone did not result in long-term survival. During the last two decades, new treatment protocols combining cytoreductive surgery with perioperative intraperitoneal and intravenous cancer chemotherapy have demonstrated very encouraging clinical results. This paper aims to clarify the pharmacologic base underlying these treatment regimens. Materials and Methods. A review of the current pharmacologic data regarding these perioperative chemotherapy protocols was undertaken. Conclusions. There is a clear pharmacokinetic and pharmacodynamic rationale for perioperative intraperitoneal and intravenous cancer chemotherapy in peritoneal surface malignancy patients.

\section{Introduction}

The peritoneal surface is an established failure site for digestive and gynecological malignancies as well as the primary location for some tumors [1-7]. Historical attempts at cure with medical therapy alone have never resulted in longterm survival. During the last two decades, new treatment modalities combining extensive cytoreductive surgery (CRS) and perioperative intraperitoneal and intravenous cancer chemotherapy have emerged. In several phase II and phase III trials, these new therapeutic approaches for peritoneal surface malignancy have shown very promising clinical results [8-18]. Although further clinical trials are mandatory, another route of exploration is equally important for further improvement of these combined treatment regimens. Pharmacologic studies of perioperative cancer chemotherapy should guide further progress in this field and offer clues for a more standardization. This paper aims to review the current pharmacologic data and point out areas of controversy needing clarification.

\section{Dose Intensification}

Dose intensification between the peritoneal compartment and the body compartment is the basic underlying pharmacologic rationale for all intraperitoneal therapy as initially stated by Dedrick et al. [19, 20]. The two above-mentioned compartments are separated by a semi permeable membrane the peritoneum. In 1941, Baron reported an elaborated description of the ultrastructure of the peritoneum in man [21]. The peritoneum consists of a monolayer of mesothelial cells supported by a basement membrane and five layers of connective tissue which account for a total thickness of $90 \mu \mathrm{m}$. The connective tissue layers include interstitial cells and a matrix of collagen, hyaluronan, and proteoglycans. The cellular component consists of fibroblasts, pericytes, parenchymal cells, and blood capillaries. Contrary to intuitive thinking, it is not the mesothelial lining which is the main transport barrier. Flessner et al. demonstrated in a rodent model that neither removal of the stagnant fluid layer on the mesothelium nor removal of the mesothelial 
lining influenced the transport over the barrier [22]. This has been confirmed in human studies in patients undergoing partial or total peritonectomy showing that the clearance of mitomycin $\mathrm{C}$ was not significantly changed by the removal of the mesothelium $[23,24]$. Basic research suggests that rather the blood capillary wall and the surrounding interstitium are the most important barriers for transport from the peritoneal space to the plasma [25]. Fluid enters the vascular compartment by diffusion from the peritoneal compartment or by absorption through the peritoneal lymphatic stomata which are concentrated on the diaphragmatic surface [26, 27]. Diffusion of fluid through the parietal peritoneum generally results in flow to the plasma compartment. Drainage through the visceral peritoneum covering the surfaces of liver, spleen, stomach, small and large bowel, and mesentery is into the portal venous blood [28].

The two-compartment Dedrick model of intraperitoneal chemotherapy is shown in Figure 1. A simplified mathematical formula describes the transport as follows: rate of mass transfer $=\mathrm{PA}\left(C_{P}-C_{B}\right)$, where $\mathrm{PA}=$ permeability area $(\mathrm{PA}=$ effective contact area $x$ permeability), $C_{P}=$ concentration in peritoneal cavity, and $C_{B}=$ concentration in the blood [29]. This formula indicates the importance of the size of the effective contact area of the peritoneal membrane. One should keep in mind that although the equation permits calculation of the pharmacokinetic advantage, the model does not predict the actual penetration of the cancer chemotherapy drug into the tissue or tumor nodule [30]. It neither predicts the value of the effective contact area. It simply describes the transfer between two compartments.

\section{Drugs Used in Perioperative Cancer Chemotherapy Protocols}

Table 1 provides an overview of drugs commonly used in perioperative cancer chemotherapy protocols and their main pharmacologic characteristics.

3.1. Mitomycin C. Mitomycin C is an alkylating antibiotic whose most important mechanism of action is through DNA cross-linking. Although mitomycin C is not regarded as a prodrug, it is not active against cancerous tissue as the unchanged molecule. The drug is modified as it enters the cell into an active state [34]. It has been used extensively in intraperitoneal cancer chemotherapy treatment protocols in appendiceal, gastric, and colorectal peritoneal carcinomatosis (PC) patients $[8,24,35,36]$. Barlogie et al. suggested in vitro thermal enhancement of mitomycin $\mathrm{C}$ [37]. Controversies still exist regarding the proper dosimetry of the chemotherapy solution. Some institutions use a single dose of mitomycin $\mathrm{C}$, others a double dose, and still others triple dose the drug over a 90-minute time period [38-40]. A remarkable difference in drug dosimetry between different groups of investigators is reported. Van Ruth and coworkers at the Dutch Cancer Institute reported a dose-finding study [40]. Their data suggest that a dose of $35 \mathrm{mg} / \mathrm{m}^{2}$ resulted in the highest peritoneal/plasma area under the curve (AUC) ratio with acceptable toxicity. In order to maintain the concentration throughout the 90 minutes perfusion time,

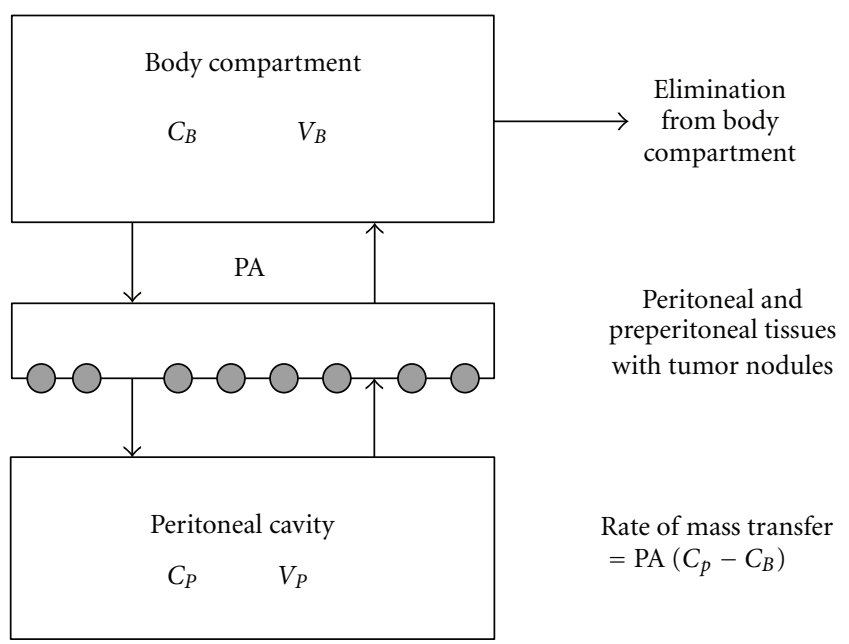

FIGURE 1: Traditional two-compartment model of peritoneal transport in which transfer of a drug from the peritoneal cavity to the blood occurs across the "peritoneal membrane." The permeabilityarea product (PA) governs this transfer and can be calculated by measuring the rate of drug disappearance from the cavity and dividing by the overall concentration difference between the peritoneal cavity and the blood (or plasma). $C_{B}$ : the free drug concentration in the blood (or plasma); $V_{B}$ : volume of distribution of the drug in the body; $C_{P}$ : the free drug concentration in the peritoneal fluid; $V_{P}$ : volume of the peritoneal cavity. Modified from R. L. Dedrick, M. F. Flessner: pharmacokinetic problems in peritoneal drug administration: Tissue penetration and surface exposure [31].

TABLE 1: Molecular weight and area under the curve ratios of intraperitoneal exposure to systemic exposure of chemotherapeutic agents used to treat peritoneal carcinomatosis.

\begin{tabular}{lcc}
\hline Drug & $\begin{array}{c}\text { Molecular weight } \\
\text { (Daltons) }\end{array}$ & $\begin{array}{c}\text { Area under the curve } \\
\text { ratio }\end{array}$ \\
\hline 5-Fluorouracil & 130.08 & 250 \\
Carboplatin & 371.25 & 10 \\
Cisplatin & 300.1 & 7.8 \\
Docetaxel & 861.9 & 552 \\
Doxorubicin & 579.99 & 230 \\
Etoposide & 588.58 & 65 \\
Floxuridine & 246.2 & 75 \\
Gemcitabine & 299.5 & 500 \\
Irinotecan & 677.19 & $\mathrm{~N} / \mathrm{A}$ \\
Melphalan & 305.2 & 93 \\
Mitomycin C & 334.3 & 23.5 \\
Mitoxantrone & 517.41 & $115-255$ \\
Oxaliplatin & 397.3 & 16 \\
Paclitaxel & 853.9 & 1000 \\
Pemetrexed & 597.49 & 40.8 \\
\hline
\end{tabular}

the dose was divided into three fractions: $50 \%$ at the start, $25 \%$ after 30 minutes, and $25 \%$ at 60 minutes. The toxicity profile of mitomycin $\mathrm{C}$, including anastomotic dehiscence and impaired wound healing, has been well characterized 
[24, 41-43]. Our data suggest large amounts of mitomycin C (62\%) remain within the body compartment after the 90minute hyperthermic intraperitoneal treatment [24].

3.2. Cisplatin. Cisplatin (cis-diamminedichloroplatinum-III CDDP) causes apoptotic cell death by formation of DNA adducts [44]. It has been well studied in the setting of adjuvant intraperitoneal chemotherapy of residual small volume ovarian cancer after CRS. Three randomized trials showed a significant survival benefit [45-47]. In the setting of cytoreductive surgery and hyperthermic intraperitoneal peroperative chemotherapy (HIPEC), cisplatin has been used for intracavitary therapy of ovarian cancer, gastric cancer, and peritoneal mesothelioma. Urano and coworkers showed an excellent in vitro and in vivo thermal augmentation of cisplatin [48].

3.3. Oxaliplatin. Oxaliplatin (oxalato-1,2-diaminocyclohexane-platinum(II)) is a third generation platinum complex with a similar cytotoxic mechanism as cisplatinum. In contrast with cisplatin, it has a proven activity in colorectal and appendiceal malignancies [49]. Its clinical use in PC patients as a component of bidirectional intraoperative chemotherapy has been pioneered by Elias and Sideris [50]. In a dose escalation and pharmacokinetic study, they showed that $460 \mathrm{mg} / \mathrm{m}^{2}$ of oxaliplatin in $2 \mathrm{~L} / \mathrm{m}^{2}$ of chemotherapy solution over 30 minutes was well tolerated [51]. The low AUC ratio is compensated by the rapid absorption of the drug into the tissue. In contrast to cisplatin and mitomycin, oxaliplatin is not stable in chloride-containing solutions and can only be administered in dextrose 5\% [52]. This may result in serious electrolyte disturbances and hyperglycemia during the intracavitary therapy [53].

A recent murine pharmacokinetic study with oxaliplatin confirmed its substantial heath augmentation [54].

3.4. Carboplatin. Carboplatin ((1,1-cyclobutanedicarboxylate)platinum(II)) is a higher molecular weight platinum compound than cisplatin which at the present time is mostly used in normothermic intraperitoneal chemotherapy protocols in patients with advanced ovarian cancer. Cjezka et al. in a clinical study with normothermic carboplatin reported a relative bioavailability (calculated as AUC values) which was at least 6-times higher in the intraperitoneal fluid than in the serum for 48 hours [55]. Los and coworkers compared carboplatin and cisplatin after intraperitoneal administration in a rat model of peritoneal carcinomatosis [56]. Their data demonstrate that despite a clear pharmacokinetic advantage of carboplatin over cisplatin, its capacity to penetrate into peritoneal cancer nodules and tumor cells is far lower than that of cisplatin. These data limit its clinical application.

3.5. Doxorubicin. Doxorubicin $\left(\mathrm{C}_{27} \mathrm{H}_{29} \mathrm{NO}_{11}\right)$ or hydroxyldaunorubicin (adriamycin) is an anthracycline antibiotic. Although being categorized as a DNA-intercalating drug, the actual mechanism of action is a critical interaction of doxorubicin with the cell surface membrane $[57,58]$. Because of its wide in vitro and in vivo activity against a broad range of malignancies, its slow clearance from the peritoneal compartment due to the high molecular weight of the hydrochloride salt $(579,99$ Dalton), its favorable area under the curve ratio of intraperitoneal to intravenous concentration times of 230, and the absence of risk for dose-limiting cardiotoxicity when used as a single-shot intraperitoneal instillation, doxorubicin was considered a potential beneficial agent for perioperative intraperitoneal delivery. This was supported by both experimental and clinical pharmacokinetic data [59-64].

3.6. Gemcitabine. Gemcitabine (2', $2^{\prime}$-difluorodeoxycitidine) is a pyrimidine analogue with a wide range of in vitro cytotoxic activity, particularly against pancreatic cancer. Pestiau et al. investigated the pharmacokinetics and tissue distribution of intraperitoneal gemcitabine in a rat model [65]. The AUC ratio (intraperitoneal/intravenous) after intraperitoneal administration was $26.8 \pm 5.8$ and as such favorable for intraperitoneal administration. Several investigators explored the use of normothermic intraperitoneal gemcitabine in advanced cancer outside the setting of cytoreductive surgery [66-68]. Resected advanced pancreatic cancer with high risk of recurrence in the operative field is a potential indication for intraoperative intraperitoneal administration of heated gemcitabine in an adjuvant setting [69].

3.7. Melphalan. Melphalan (L-phenylalanine mustard) is a chemotherapy drug belonging to the class of nitrogen mustard alkylating agents. Alberts et al. were the first to investigate the pharmacokinetics of intraperitoneal melphalan [70]. Melphalan systemic absorption from the peritoneal cavity averaged only $39 \%$ of the administered dose. Urano showed a remarkable heat augmentation of melphalan [48]. Glehen and coworkers investigated the effect of hyperthermia on the pharmacokinetics of intraperitoneal melphalan in a rat model [71]. Hyperthermia decreased the AUC of peritoneal fluid without increasing the plasma AUC. Intraabdominal tissue concentrations were markedly elevated compared to normothermic controls. Sugarbaker et al. in a pharmacokinetic and phase-II study of intraoperative intraperitoneal melphalan showed that $90 \%$ of the cancer chemotherapy drug was absorbed during the 90-minute procedure with a 30-times higher exposure at the peritoneal surface than in the blood [72]. Concentrations in tumor nodules were 10-times higher than concentrations in the blood. This favorable pharmacokinetic profile and tissue distributions, combined with cytotoxic activity against a wide range of malignancies, makes melphalan an excellent salvage drug for intraperitoneal treatment protocols.

3.8. Taxanes. Paclitaxel and docetaxel are taxanes considered for i.p. chemotherapy. The taxanes stabilize the microtubule against depolymerization, thereby disrupting normal microtubule dynamics [73]. They exert cytotoxic activity against a broad range of tumors. Due to their high molecular weight these molecules have a remarkable high AUC ratio of 853 and 861 respectively, [74]. This translates itself into a clear pharmacokinetic advantage for intraperitoneal administration [75]. The data regarding possible thermal augmentation of taxanes are conflicting [76-79]. Taxanes 
have been used in a neoadjuvant intraperitoneal setting as well as intraoperatively and postoperatively. Postoperative intraperitoneal paclitaxel conferred a survival benefit in this postoperative setting. Their cell-cycle specific mechanism of action makes them a particular good candidate for repetitive application such as in early postoperative intraperitoneal chemotherapy (EPIC) or normothermic adjuvant postoperative intraperitoneal chemotherapy [45, 46, 80-82].

3.9. 5-Fluorouracil. 5-Fluorouracil is an inhibitor of thymidylate synthase. Since thymidine is the only nucleotide precursor specific to DNA, thymidilate synthase is an obvious target for cytotoxic agents. 5-Fluorouracil is intracellularly metabolized in two steps to its active metabolite, 5-fluoro2 -deoxyuridine monophosphate (FdUMP). This molecule will, in the presence of reduced folate, bind at the same site and with the same affinity as deoxyuridine monophosphate (dUMP) and ultimately impair the enzymatic activity of the thymidilate synthetase [83]. The action of 5-fluorouracil is therefore cell cycle specific. Also 5-FU by its metabolites 5-fluoro-uridine diphosphate and 5-fluoro-uridine triphosphate gets incorporated in RNA, resulting in a second cytotoxic pathway. Minor augmentation of 5-fluorouracil by mild hyperthermia is reported $[84,85]$. 5-Fluorouracil is not chemically compatible with other drugs in a mixed solution for infusion or instillation. These characteristics limit the use of 5-fluorouracil perioperatively to either early postoperative intraperitoneal chemotherapy or intraoperative intravenous 5-fluorouracil.

3.10. Pemetrexed. Pemetrexed is a multitargeted antifolate. It is an analogue of folic acid with cytotoxic activity against a variety of malignancies, especially mesothelioma and colon cancer. It belongs to the antimetabolites. It acts mainly as a thymidilate synthase inhibitor but is also unique in terms of cellular transport and lipid solubility [86]. Pestieau et al. reported favorable intraperitoneal pharmacokinetics [87]. It is currently under investigation for the intraperitoneal treatment of peritoneal mesothelioma.

3.11. Ifosfamide. Ifosfamide is a prodrug which needs the cytochrome P 450 system of liver or red blood cells to be activated to its active metabolite 4-hydroxyifosfamide. Consequently, it requires intravenous administration rather than intraperitoneal instillation for its cytotoxic activity. It is one of four drugs that show true heat synergy, with 5- to 10-times the duration of tumor control with $41.5^{\circ} \mathrm{C}$ heat as compared to normal temperatures [48]. It may be an ideal systemic drug to increase the cytotoxicity of hyperthermic intraperitoneal peroperative chemotherapy. Our pharmacokinetic data show the presence of ifosfamide and its active metabolite in peritoneal tumor nodules after intravenous continuous infusion during bidirectional intraoperative chemotherapy. In these bidirectional treatment protocols, intravenous and intraperitoneal routes of administration are combined after CRS inside the operating room.
TABLE 2: Pharmacokinetic and pharmacodynamic variables involved in the administration of perioperative cancer chemotherapy in peritoneal surface malignancy patients.

\begin{tabular}{lc}
\hline Pharmacokinetic variables & Pharmacodynamic variables \\
\hline Dose & Temperature \\
Volume & Nodule size of residual tumor \\
Duration & Density \\
Carrier solution & Binding \\
Pressure & Interstitial fluid pressure \\
Vasoactive agents & Charge \\
Macromolecular vehicles & Vascularity \\
\hline
\end{tabular}

\section{Pharmacologic Variables in Perioperative Chemotherapy}

Pharmacokinetics describe what the body does to the drug, whereas pharmacodynamics describe what the drug does to the body. Table 2 summarizes the pharmacokinetic and pharmacodynamic variables involved in perioperative intraperitoneal and intravenous chemotherapy. One of the most challenging problems hindering a further wide application of these new treatment modalities is the compelling variety of regimens available worldwide. These protocols are sometimes based on little or no pharmacologic data. Furthermore this variability in dosimetry and technology makes multicenter registry or trials very difficult. The international scientific community must come up with a consensus on standardizing the application. This should be based on a thorough review of the available pharmacologic data and clinical results.

\section{Pharmacologic Controversies}

5.1. Concentration-Based or Body Surface Area-(BSA-) Based Dosimetry. Most groups use a drug dose based on calculated body surface area $\left(\mathrm{mg} / \mathrm{m}^{2}\right)$. However, Rubin et al. demonstrate that there is an imperfect correlation between actual peritoneal surface area and calculated body surface area and there may be sex differences in peritoneal surface areas, which in turn affects absorption characteristics [88]. The female has a $10 \%$ larger peritoneal surface in proportion to body size than the male. There have been attempts to estimate the functional peritoneal surface area through applying stereological methods to computer tomography (CT) scans by extrapolating data from cadaver measurements $[89,90]$. Body surface area is an accurate predictor of drug metabolism and is useful for estimating systemic drug toxicity.

Some groups use a totally different dosimetry regimen based on concentration. The total amount of cancer chemotherapy is mixed in a large volume of carrier solution (usually six liters) that is placed in a reservoir. For example, Deraco and Rossi at the Milan Cancer Institute use doxorubicin $15.25 \mathrm{mg} / \mathrm{m}^{2} / \mathrm{L}$ and cisplatin $43 \mathrm{mg} / \mathrm{m}^{2} / \mathrm{L}$ with a total volume of 6 liters. Glehen and Gilly from Lyon have used mitomycin C $0.5 \mathrm{mg} / \mathrm{kg}$ and cisplatin $0.7 \mathrm{mg} / \mathrm{kg}$ in a total volume of 4 to 6 liters [91-94]. In this closed method, the amount of 
chemotherapy solution in contact with the peritoneal surface is determined by multiple variables: the amount of distention (between 2 and 6 liters) of the abdominal cavity, which is induced by the chemotherapy solution, the patient's sex, the amount of ascites present preoperatively, and the extent of the visceral resection. The big advantage of a concentrationbased system is that the residual tumor nodules after CRS are exposed to a constant diffusional force and thus cytotoxicity. Unfortunately, the prize to be paid for a better prediction of the efficacy of the intraperitoneal chemotherapy is a high unpredictability of the plasmatic cancer chemotherapy levels and thus toxicity. Indeed, according to the above-mentioned Dedrick formula of transport over the peritoneal membrane, an increase in the volume of intraperitoneal chemotherapy solution will cause an increase in both diffusion surface and the amount of drug transferred from peritoneal space to plasma. For example, in 10 patients dialyzed with different volumes ranging from 0.5 up to 3 liters, there is a linear rise in mass transfer [95].

Other factors contribute to the controversy over the proper dosage of chemotherapy solution. Some institutions use a single dose of the intraperitoneal drug; others use a double, or even triple, dose of the same drug over a 90minute period [96-98].

5.2. Pharmacokinetics versus Pharmacodynamics. Until recently, the pharmacologic efficacy of intraperitoneal cancer chemotherapy protocols was assessed by looking at the pharmacokinetics of the i.p. and i.v. compartments. The efficacy of the IP protocol was then quantified by calculating the area-under-the-curve (AUC) ratio of the IP exposure over the AUC of the IV exposure. This, however, does not take into account any pharmacodynamic variables. Figure 2 demonstrates that the pharmacodynamic event of doxorubicin binding to the tumor nodule results in higher intratumoral concentrations than can be predicted by the simple IP/IV pharmacokinetics [32]. Another example of the equal importance of pharmacodynamics is shown in Figure 3. With identical pharmacokinetics the amount of doxorubicin showing up in the less dense diffuse peritoneal adenomysis (DPAM) subtype of appendiceal malignancy PC is statistically significantly lower than in the more dense peritoneal mucinous carcinomatosis (PMCA) nodules [32]. The identical pharmacokinetic advantage (expressed as AUC IP/IV ratios) resulted in different drug levels according to the density of the tumor nodules; this stressed the importance of pharmacodynamic variables such as tumor nodule density, size, and vascularity. Increased awareness of the pharmacodynamic aspects of these treatment protocols has also been reported by Ceelen et al. [99]. Therefore, it was proposed that the tumor nodule was a more appropriate pharmacological endpoint than AUC ratios.

5.3. Adding Intravenous Intraoperative Chemotherapy to the Equation. By combining intraoperative intravenous and intraoperative intraperitoneal cancer chemotherapy, a bidirectional diffusion gradient is created through the intermediate tissue layer which contains the cancer nodules. This offers opportunities for optimizing cancer chemotherapy

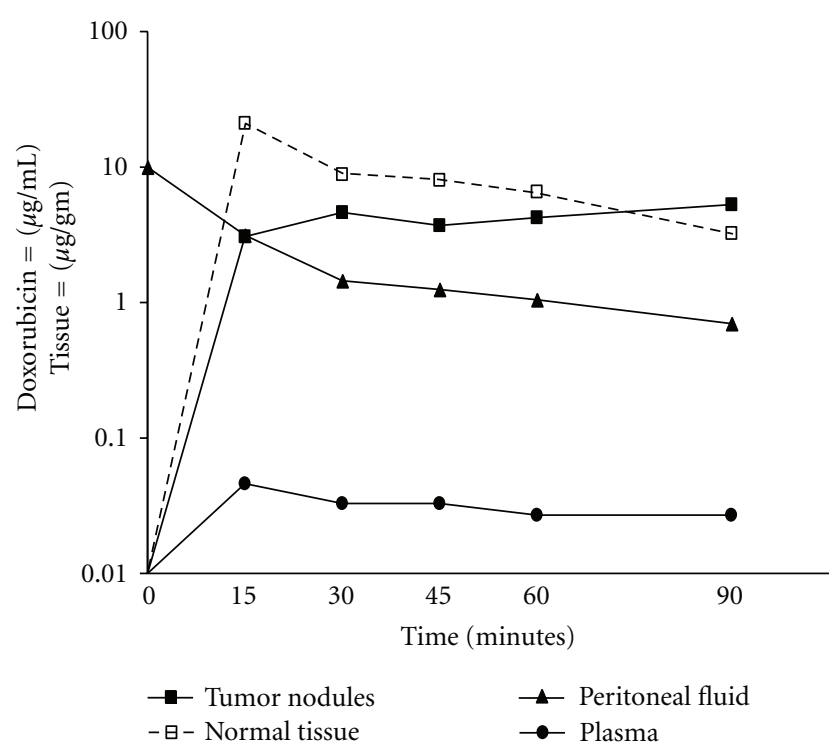

FIGURE 2: Doxorubicin concentration in plasma, peritoneal fluid, tumor nodules, and normal adjacent tissues [32].

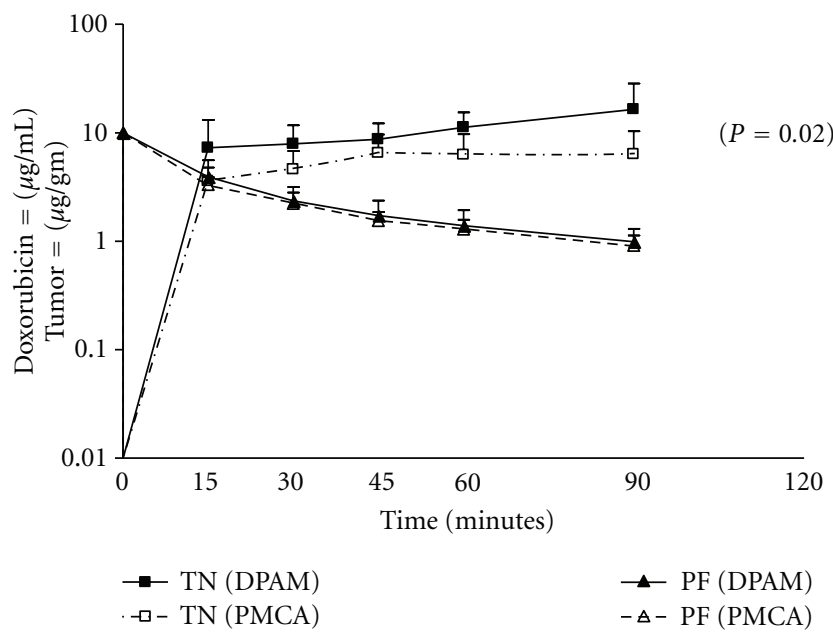

FIGURE 3: Doxorubicin levels in appendiceal tumor tissue showing diffuse peritoneal adenomucinosis (DPAM) versus peritoneal mucinous carcinomatosis (PMCA). Peritoneal fluid concentrations are also shown. TN: tumor nodule; PF: peritoneal fluid [32].

delivery to the target peritoneal tumor nodules. In 2002, Elias et al. first reported the clinical use of intraoperative intravenous 5-fluorouracil and leucovorin in conjunction with oxaliplatin-based hyperthermic intraperitoneal perioperative chemotherapy [100]. Figure 4 demonstrates the concentrations of 5-fluorouracil in tumor nodules that were harvested during bidirectional (intraperitoneal doxorubicin and mitomycin $\mathrm{C}$ plus rapid infusion intravenous 5fluorouracil) intraoperative chemotherapy treatment [33]. The rapid distribution of the 5-fluorouracil after IV administration affects all compartments similarly. The metabolism of the 5-fluorouracil on the other hand is mainly restricted 


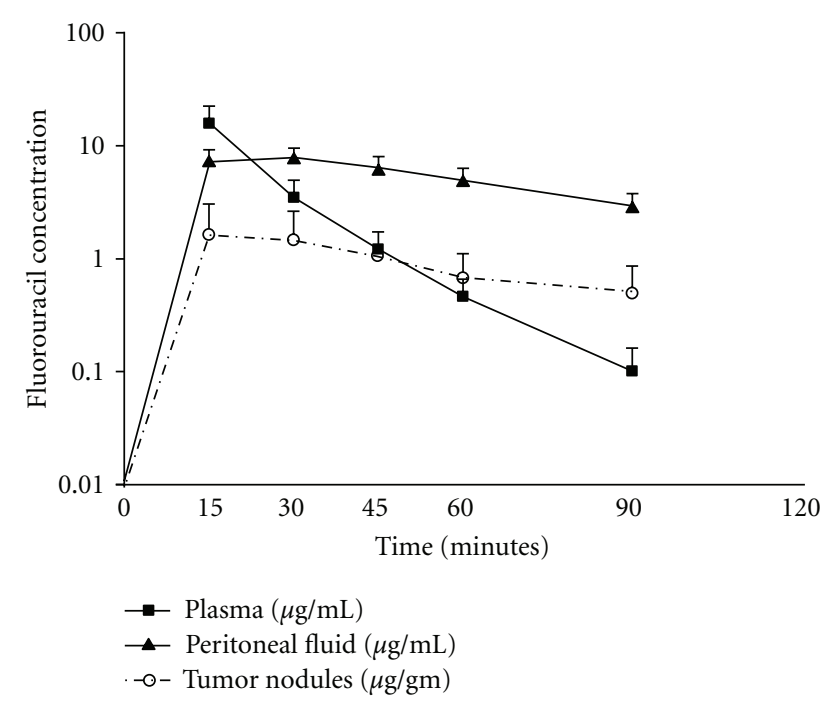

FIgURE 4: 5-Fluorouracil concentrations in plasma, peritoneal fluid, and tumor nodules after intravenous administration during hyperthermic intraperitoneal chemotherapy procedure [33].

to the plasma compartment by the liver. The high level of 5fluorouracil persists within the peritoneal fluid because the drug can only leave the peritoneal space by back diffusion through the peritoneal and subperitoneal tissues; the enzyme dihydropyrimidine dehydrogenase is not present in the artificial ascites fluid. These data show clear pharmacokinetic advantage for the intraoperative intravenous administration of 5-fluorouracil. Although 5-fluorouracil is administered as a normothermic intravenous solution, it penetrates into the heated tumor nodules. Normothermic administered 5-fluorouracil becomes subject to augmentation of mild hyperthermia of the subperitoneal compartment. Therefore, heat targeting is achieved by modulating the timing of intravenous chemotherapy.

Recently, we were able to demonstrate a similar pharmacokinetic advantage and heat targeting of intraoperative intravenous ifosfamide (continuous infusion over $90 \mathrm{~min}$ utes) [101].

\section{Conclusions}

The last two decades saw the emergence of perioperative cancer chemotherapy protocols in the treatment of PC patients. This has resulted in remarkable clinical successes in contrast with prior failures. Now that the concept is proven, time has come to further improve the treatment protocols. Building more pharmacologic data on perioperative chemotherapy in PC patients should result in both more standardization and better clinical outcome.

\section{References}

[1] D. G. Jayne, S. Fook, C. Loi, and F. Seow-Choen, "Peritoneal carcinomatosis from colorectal cancer," British Journal of Surgery, vol. 89, no. 12, pp. 1545-1550, 2002.
[2] J. T. Brodsky and A. M. Cohen, "Peritoneal seeding following potentially curative resection of colonic carcinoma: implications for adjuvant therapy," Diseases of the Colon and Rectum, vol. 34, no. 8, pp. 723-727, 1991.

[3] D. Z. J. Chu, N. P. Lang, C. Thompson, P. K. Osteen, and K. C. Westbrook, "Peritoneal carcinomatosis in nongynecologic malignancy. A prospective study of prognostic factors," Cancer, vol. 63, no. 2, pp. 364-367, 1989.

[4] B. Sadeghi, C. Arvieux, O. Glehen et al., "Peritoneal carcinomatosis from non-gynecologic malignancies: results of the EVOCAPE 1 multicentric prospective study," Cancer, vol. 88, no. 2, pp. 358-363, 2000.

[5] N. A. Shepherd, K. J. Baxter, and S. B. Love, "The prognostic importance of peritoneal involvement in colonic cancer: a prospective evaluation," Gastroenterology, vol. 112, no. 4, pp. 1096-1102, 1997.

[6] A. Jemal, T. Murray, E. Ward, and A. Samuels, "Cancer statistics, 2005," Ca-A Cancer Journal for Clinicians, vol. 55, pp. 10-30, 2005.

[7] A. Poveda, "Ovarian cancer treatment: what is new," International Journal of Gynecological Cancer, vol. 13, no. 2, pp. 241-250, 2003.

[8] V. J. Verwaal, S. Van Ruth, E. De Bree et al., "Randomized trial of cytoreduction and hyperthermic intraperitoneal chemotherapy versus systemic chemotherapy and palliative surgery in patients with peritoneal carcinomatosis of colorectal cancer," Journal of Clinical Oncology, vol. 21, no. 20, pp. 3737-3743, 2003.

[9] V. J. Verwaal, S. Bruin, H. Boot, G. van Slooten, and H. van Tinteren, "8-Year follow-up of randomized trial: cytoreduction and hyperthermic intraperitoneal chemotherapy versus systemic chemotherapy in patients with peritoneal carcinomatosis of colorectal cancer," Annals of Surgical Oncology, vol. 15, no. 9, pp. 2426-2432, 2008.

[10] D. Elias, J. H. Lefevre, J. Chevalier et al., "Complete cytoreductive surgery plus intraperitoneal chemohyperthermia with oxaliplatin for peritoneal carcinomatosis of colorectal origin," Journal of Clinical Oncology, vol. 27, no. 5, pp. 681685, 2009.

[11] O. Glehen, F. Kwiatkowski, P. H. Sugarbaker et al., "Cytoreductive surgery combined with perioperative intraperitoneal chemotherapy for the management of peritoneal carcinomatosis from colorectal cancer: a multi-institutional study," Journal of Clinical Oncology, vol. 22, no. 16, pp. 3284-3292, 2004.

[12] O. Glehen, F. Mithieux, D. Osinsky et al., "Surgery combined with peritonectomy procedures and intraperitoneal chemohyperthermia in abdominal cancers with peritoneal carcinomatosis: a phase II study," Journal of Clinical Oncology, vol. 21, no. 5, pp. 799-806, 2003.

[13] T. D. Yan, D. Black, P. H. Sugarbaker et al., "A systematic review and meta-analysis of the randomized controlled trials on adjuvant intraperitoneal chemotherapy for resectable gastric cancer," Annals of Surgical Oncology, vol. 14, no. 10, pp. 2702-2713, 2007.

[14] L. Bijelic, A. Jonson, and P. H. Sugarbaker, "Systematic review of cytoreductive surgery and heated intraoperative intraperitoneal chemotherapy for treatment of peritoneal carcinomatosis in primary and recurrent ovarian cancer," Annals of Oncology, vol. 18, no. 12, pp. 1943-1950, 2007.

[15] T. D. Yan, L. Welch, D. Black, and P. H. Sugarbaker, "A systematic review on the efficacy of cytoreductive surgery combined with perioperative intraperitoneal chemotherapy 
for diffuse malignancy peritoneal mesothelioma," Annals of Oncology, vol. 18, no. 5, pp. 827-834, 2007.

[16] T. D. Yan, D. Black, R. Savady, and P. H. Sugarbaker, "A systematic review on the efficacy of cytoreductive surgery and perioperative intraperitoneal chemotherapy for pseudomyxoma peritonei," Annals of Surgical Oncology, vol. 14, no. 2, pp. 484-492, 2007.

[17] T. D. Yan, D. Black, R. Savady, and P. H. Sugarbaker, "Systematic review on the efficacy of cytoreductive surgery combined with perioperative intraperitoneal chemotherapy for peritoneal carcinomatosis from colorectal carcinoma," Journal of Clinical Oncology, vol. 24, no. 24, pp. 4011-4019, 2006.

[18] D. Elias, O. Glehen, and F. Gilly, "Carcinose péritonéales d'origine digestive et primitive," in Proceedings of the Rapport du 110 éme Congrès de l'AFC, Arnette : Wolters Kluwer France, 2008.

[19] R. L. Dedrick, C. E. Myers, P. M. Bungay, and V. T. Devita, "Pharmacokinetic rationale for peritoneal drug administration in the treatment of ovarian cancer," Cancer Treatment Reports, vol. 62, no. 1, pp. 1-11, 1978.

[20] R. L. Dedrick, "Theoretical and experimental bases of intraperitoneal chemotherapy," Seminars in Oncology, vol. 12, no. 3, supplement 4, pp. 1-6, 1985.

[21] M. A. Baron, "Structure of the intestinal peritoneum in man," American Journal of Anatomy, vol. 69, pp. 439-497, 1941.

[22] M. Flessner, J. Henegar, S. Bigler, and L. Genous, "Is the peritoneum a significant transport barrier in peritoneal dialysis?" Peritoneal Dialysis International, vol. 23, no. 6, pp. 542-549, 2003.

[23] V. De Lima Vazquez, O. A. Stuart, F. Mohamed, and P. H. Sugarbaker, "Extent of parietal peritonectomy does not change intraperitoneal chemotherapy pharmacokinetics," Cancer Chemotherapy and Pharmacology, vol. 52, no. 2, pp. 108-112, 2003.

[24] K. Van der Speeten, O. A. Stuart, D. Chang, H. Mahteme, and P. H. Sugarbaker, "Changes induced by surgical and clinical factors in the pharmacology of intraperitoneal mitomycin $\mathrm{C}$ in 145 patients with peritoneal carcinomatosis," Cancer Chemotherapy and Pharmacology, vol. 68, no. 1, pp. 147-156, 2011.

[25] G. Stelin and B. Rippe, "A phenomenological interpretation of the variation in dialysate volume with dwell time in CAPD," Kidney International, vol. 38, no. 3, pp. 465-472, 1990.

[26] U. Bettendorf, "Lymph flow mechanism of the subperitoneal diaphragmatic lymphatics," Lymphology, vol. 11, no. 3, pp. 111-116, 1978.

[27] U. Bettendorf, "Electronmicroscopic studies on the peritoneal resorption of intraperitoneally injected latex particles via the diaphragmatic lymphatics," Lymphology, vol. 12, no. 2, pp. 66-70, 1979.

[28] M. H. Katz and R. M. Barone, "The rationale of perioperative intraperitoneal chemotherapy in the treatment of peritoneal surface malignancies," Surgical Oncology Clinics of North America, vol. 12, no. 3, pp. 673-688, 2003.

[29] R. L. Dedrick, C. E. Myers, P. M. Bungay, and V. T. Devita, "Pharmacokinetic rationale for peritoneal drug administration in the treatment of ovarian cancer," Cancer Treatment Reports, vol. 62, no. 1, pp. 1-11, 1978.

[30] M. F. Flessner, J. D. Fenstermacher, R. L. Dedrick, and R. G. Blasberg, "A distributed model of peritoneal-plasma transport: tissue concentration gradients," American Journal of Physiology, vol. 248, no. 3, pp. F425-F435, 1985.
[31] R. L. Dedrick and M. F. Flessner, "Pharmacokinetic problems in peritoneal drug administration: tissue penetration and surface exposure," Journal of the National Cancer Institute, vol. 89, no. 7, pp. 480-487, 1997.

[32] K. Van der Speeten, O. A. Stuart, H. Mahteme, and P. H. Sugarbaker, "A pharmacologic analysis of intraoperative intracavitary cancer chemotherapy with doxorubicin," Cancer Chemotherapy and Pharmacology, vol. 63, no. 5, pp. 799805, 2009.

[33] K. Van der Speeten, O. A. Stuart, H. Mahteme, and P. H. Sugarbaker, "Pharmacology of perioperative 5-fluorouracil," Journal of Surgical Oncology, vol. 102, no. 7, pp. 730-735, 2010.

[34] N. R. Bachur, S. L. Gordon, M. V. Gee, and H. Kon, "NADPH cytochrome P-450 reductase activation of quinone anticancer agents to free radicals," Proceedings of the National Academy of Sciences of the United States of America, vol. 76, no. 2, pp. 954-957, 1979.

[35] D. Elias, P. Dubé, F. Blot et al., "Peritoneal carcinomatosis treatment with curative intent: the Institut Gustave-Roussy experience," European Journal of Surgical Oncology, vol. 23, no. 4, pp. 317-321, 1997.

[36] P. H. Sugarbaker and D. Chang, "Results of treatment of 385 patients with peritoneal surface spread of appendiceal malignancy," Annals of Surgical Oncology, vol. 6, no. 8, pp. 727-731, 1999.

[37] B. Barlogie, P. M. Corry, and B. Drewinko, "In vitro thermochemotherapy of human colon cancer cells with cisdichlorodiammineplatinum(II) and mitomycin C," Cancer Research, vol. 40, no. 4, pp. 1165-1168, 1980.

[38] P. H. Sugarbaker, "Successful management of microscopic residual disease in large bowel cancer," Cancer Chemotherapy and Pharmacology, Supplement, vol. 43, pp. S15-S25, 1999.

[39] J. Esquivel, "Technology of hyperthermic intraperitoneal chemotherapy in the United States, Europe, China, Japan, and Korea," Cancer Journal, vol. 15, no. 3, pp. 249-254, 2009.

[40] S. Van Ruth, V. J. Verwaal, and F. A. N. Zoetmulder, "Pharmacokinetics of intraperitoneal mitomycin C," Surgical Oncology Clinics of North America, vol. 12, no. 3, pp. 771780, 2003.

[41] R. M. Smeenk, V. J. Verwaal, and F. A. N. Zoetmulder, "Toxicity and mortality of cytoreduction and intraoperative hyperthermic intraperitoneal chemotherapy in pseudomyxoma peritonei-a report of 103 procedures," European Journal of Surgical Oncology, vol. 32, no. 2, pp. 186-190, 2006.

[42] O. Glehen, D. Osinsky, E. Cotte et al., "Intraperitoneal chemohyperthermia using a closed abdominal procedure and cytoreductive surgery for the treatment of peritoneal carcinomatosis: morbidity and mortality analysis of 216 consecutive procedures," Annals of Surgical Oncology, vol. 10, no. 8, pp. 863-869, 2003.

[43] P. H. Sugarbaker, R. Alderman, G. Edwards et al., "Prospective morbidity and mortality assessment of cytoreductive surgery plus perioperative intraperitoneal chemotherapy to treat peritoneal dissemination of appendiceal mucinous malignancy," Annals of Surgical Oncology, vol. 13, no. 5, pp. 635-644, 2006.

[44] V. Cepeda, M. A. Fuertes, J. Castilla, C. Alonso, C. Quevedo, and J. M. Pérez, "Biochemical mechanisms of cisplatin cytotoxicity," Anti-Cancer Agents in Medicinal Chemistry, vol. 7, no. 1, pp. 3-18, 2007.

[45] D. K. Armstrong, B. Bundy, L. Wenzel et al., "Intraperitoneal cisplatin and paclitaxel in ovarian cancer," New England Journal of Medicine, vol. 354, no. 1, pp. 34-43, 2006. 
[46] M. Markman, B. N. Bundy, D. S. Alberts et al., "Phase III trial of standard-dose intravenous cisplatin plus paclitaxel versus moderately high-dose carboplatin followed by intravenous paclitaxel and intraperitoneal cisplatin in small-volume stage III ovarian carcinoma: an intergroup study of the gynecologic oncology group, Southwestern Oncology Group, and Eastern Cooperative Oncology Group," Journal of Clinical Oncology, vol. 19, no. 4, pp. 1001-1007, 2001.

[47] D. S. Alberts, P. Y. Liu, E. V. Hannigan et al., "Intraperitoneal cisplatin plus intravenous cyclophosphamide versus intravenous cisplatin plus intravenous cyclophosphamide for stage III ovarian cancer," New England Journal of Medicine, vol. 335, no. 26, pp. 1950-1955, 1996.

[48] M. Urano, M. Kuroda, and Y. Nishimura, "For the clinical application of thermochemotherapy given at mild temperatures," International Journal of Hyperthermia, vol. 15, no. 2, pp. 79-107, 1999.

[49] J. H. Stewart IIII, P. Shen, G. Russell et al., "A phase I trial of oxaliplatin for intraperitoneal hyperthermic chemoperfusion for the treatment of peritoneal surface dissemination from colorectal and appendiceal cancers," Annals of Surgical Oncology, vol. 15, no. 8, pp. 2137-2145, 2008.

[50] D. M. Elias and L. Sideris, "Pharmacokinetics of heated intraoperative intraperitoneal oxaliplatin after complete resection of peritoneal carcinomatosis," Surgical Oncology Clinics of North America, vol. 12, no. 3, pp. 755-769, 2003.

[51] D. Elias, M. Bonnay, J. M. Puizillou et al., "Heated intraoperative intraperitoneal oxaliplatin after complete resection of peritoneal carcinomatosis: pharmacokinetics and tissue distribution," Annals of Oncology, vol. 13, no. 2, pp. 267-272, 2002.

[52] E. Jerremalm, M. Hedeland, I. Wallin, U. Bondesson, and H. Ehrsson, "Oxaliplatin degradation in the presence of chloride: identification and cytotoxicity of the monochloro monooxalato complex," Pharmaceutical Research, vol. 21, no. 5, pp. 891-894, 2004.

[53] F. de Somer, W. Ceelen, J. Delanghe et al., "Severe hyponatremia, hyperglycemia, and hyperlactatemia are associated with intraoperative hyperthermic intraperitoneal chemoperfusion with oxaliplatin," Peritoneal Dialysis International, vol. 28, no. 1, pp. 61-66, 2008.

[54] N. Piché, F. A. Leblond, L. Sidéris et al., "Rationale for heating oxaliplatin for the intraperitoneal treatment of peritoneal carcinomatosis: a study of the effect of heat on intraperitoneal oxaliplatin using a murine model," Annals of Surgery, vol. 254, no. 1, pp. 138-144, 2011.

[55] M. Czejka, W. Jäger, J. Schüller et al., "Pharmacokinetics of carboplatin after intraperitoneal administration," Archiv der Pharmazie, vol. 324, no. 3, pp. 183-184, 1991.

[56] G. Los, E. M. E. Verdegaal, P. H. A. Mutsaers, and J. G. McVie, "Penetration of carboplatin and cisplatin into rat peritoneal tumor nodules after intraperitoneal chemotherapy," Cancer Chemotherapy and Pharmacology, vol. 28, no. 3, pp. 159-165, 1991.

[57] T. R. Tritton, "Cell surface actions of adriamycin," Pharmacology and Therapeutics, vol. 49, no. 3, pp. 293-309, 1991.

[58] P. Lane, P. Vichi, D. L. Bain, and T. R. Tritton, "Temperature dependence studies of adriamycin uptake and cytotoxicity," Cancer Research, vol. 47, no. 15, pp. 4038-4042, 1987.

[59] P. Jacquet, A. Averbach, O. A. Stuart, D. Chang, and P. H. Sugarbaker, "Hyperthermic intraperitoneal doxorubicin: pharmacokinetics, metabolism, and tissue distribution in a rat model," Cancer Chemotherapy and Pharmacology, vol. 41, no. 2, pp. 147-154, 1998.
[60] K. Van der Speeten, O. A. Stuart, H. Mahteme, and P. H. Sugarbaker, "A pharmacologic analysis of intraoperative intracavitary cancer chemotherapy with doxorubicin," Cancer Chemotherapy and Pharmacology, vol. 63, no. 5, pp. 799805, 2009.

[61] P. B. Johansen, "Doxorubicin pharmacokinetics after intravenous and intraperitoneal administration in the nude mouse," Cancer Chemotherapy and Pharmacology, vol. 5, no. 4, pp. 267-270, 1981.

[62] R. F. Ozols, K. R. Grotzinger, and R. I. Fisher, "Kinetic characterization and response to chemotherapy in a transplantable murine ovarian cancer," Cancer Research, vol. 39, no. 8, pp. 3202-3208, 1979.

[63] R. F. Ozols, G. Y. Locker, and J. H. Doroshow, "Pharmacokinetics of adriamycin and tissue penetration in murine ovarian cancer," Cancer Research, vol. 39, no. 8, pp. 32093214, 1979.

[64] R. F. Ozols, R. C. Young, and J. L. Speyer, "Phase I and pharmacological studies of adriamycin administered intraperitoneally to patients with ovarian cancer," Cancer Research, vol. 42, no. 10, pp. 4265-4269, 1982.

[65] S. R. Pestieau, O. A. Stuart, D. Chang, P. Jacquet, and P. H. Sugarbaker, "Pharmacokinetics of intraperitoneal gemcitabine in a rat model," Tumori, vol. 84, no. 6, pp. 706-711, 1998.

[66] P. Sabbatini, C. Aghajanian, M. Leitao et al., "Intraperitoneal cisplatin with intraperitoneal gemcitabine in patients with epithelial ovarian cancer: results of a phase I/II Trial," Clinical Cancer Research, vol. 10, no. 9, pp. 2962-2967, 2004.

[67] R. J. Morgan Jr., T. W. Synold, B. Xi et al., "Phase I trial of intraperitoneal gemcitabine in the treatment of advanced malignancies primarily confined to the peritoneal cavity," Clinical Cancer Research, vol. 13, no. 4, pp. 1232-1237, 2007.

[68] T. C. Gamblin, M. J. Egorin, E. G. Zuhowski et al., "Intraperitoneal gemcitabine pharmacokinetics: a pilot and pharmacokinetic study in patients with advanced adenocarcinoma of the pancreas," Cancer Chemotherapy and Pharmacology, vol. 62, no. 4, pp. 647-653, 2008.

[69] P. H. Sugarbaker, O. A. Stuart, and L. Bijelic, "Intraperitoneal gemcitabine chemotherapy treatment for patients with resected pancreatic cancer: rationale and report of early data," International Journal of Surgical Oncology, vol. 2011, Article ID 161862, 7 pages, 2011.

[70] D. S. Alberts, H. S. Chen, S. Y. Chang, and Y. M. Peng, "The disposition of intraperitoneal bleomycin, melphalan, and vinblastine in cancer patients," Recent Results in Cancer Research, vol. 74, pp. 293-299, 1980.

[71] O. Glehen, O. A. Stuart, F. Mohamed, and P. H. Sugarbaker, "Hyperthermia modifies pharmacokinetics and tissue distribution of intraperitoneal melphalan in a rat model," Cancer Chemotherapy and Pharmacology, vol. 54, no. 1, pp. 79-84, 2004.

[72] P. H. Sugarbaker and O. A. Stuart, "Pharmacokinetic and phase II study of heated intraoperative intraperitoneal melphalan," Cancer Chemotherapy and Pharmacology, vol. 59, no. 2, pp. 151-155, 2007.

[73] W. P. Ceelen, L. Påhlman, and H. Mahteme, "Pharmacodynamic aspects of intraperitoneal cytotoxic therapy," Cancer Treatment and Research, vol. 134, pp. 195-214, 2007.

[74] P. H. Sugarbaker, J. T. Mora, P. Carmignani, O. A. Stuart, and D. Yoo, "Update on chemotherapeutic agents utilized for perioperative intraperitoneal chemotherapy," Oncologist, vol. 10, no. 2, pp. 112-122, 2005. 
[75] F. Mohamed and P. H. Sugarbaker, "Intraperitoneal taxanes," Surgical Oncology Clinics of North America, vol. 12, no. 3, pp. 825-833, 2003.

[76] R. C. Rietbroek, D. M. Katschinski, M. H. E. Reijers et al., "Lack of thermal enhancement for taxanes in vitro," International Journal of Hyperthermia, vol. 13, no. 5, pp. 525-533, 1997.

[77] D. S. Schrump, S. Zhai, D. M. Nguyen et al., "Pharmacokinetics of paclitaxel administered by hyperthermic retrograde isolated lung perfusion techniques," Journal of Thoracic and Cardiovascular Surgery, vol. 123, no. 4, pp. 686-694, 2002.

[78] A. Cividalli, G. Cruciani, E. Livdi, P. Pasqualetti, and D. Tirindelli Danesi, "Hyperthermia enhances the response of paclitaxel and radiation in a mouse adenocarcinoma," International Journal of Radiation Oncology Biology Physics, vol. 44, no. 2, pp. 407-412, 1999.

[79] F. Mohamed, O. A. Stuart, O. Glehen, M. Urano, and P. H. Sugarbaker, "Docetaxel and hyperthermia: factors that modify thermal enhancement," Journal of Surgical Oncology, vol. 88, no. 1, pp. 14-20, 2004.

[80] Y. Yonemura, E. Bandou, T. Sawa et al., "Neoadjuvant treatment of gastric cancer with peritoneal dissemination," European Journal of Surgical Oncology, vol. 32, no. 6, pp. 661-665, 2006.

[81] E. De Bree, H. Rosing, J. H. Beijnen et al., "Pharmacokinetic study of docetaxel in intraoperative hyperthermic i.p. chemotherapy for ovarian cancer," Anti-Cancer Drugs, vol. 14, no. 2, pp. 103-110, 2003.

[82] E. DeBree, H. Rosing, D. Filis et al., "Cytoreductive surgery and intraoperative hyperthermic intraperitoneal chemotherapy with paclitaxel: a clinical and pharmacokinetic study," Annals of Surgical Oncology, vol. 15, no. 4, pp. 1183-1192, 2008.

[83] J. L. Speyer, J. M. Collins, and R. L. Dedrick, "Phase I and pharmacological studies of 5-fluorouracil administered intraperitoneally," Cancer Research, vol. 40, no. 3, pp. 567$572,1980$.

[84] P. Jacquet, A. Averbach, A. D. Stephens, O. Anthony Stuart, D. Chang, and P. H. Sugarbaker, "Heated intraoperative intraperitoneal mitomycin $\mathrm{C}$ and early postoperative intraperitoneal 5-fluorouracil: pharmacokinetic studies," Oncology, vol. 55, no. 2, pp. 130-138, 1998.

[85] R. B. Diasio and Z. Lu, "Dihydropyrimidine dehydrogenase activity and fluorouracil chemotherapy," Journal of Clinical Oncology, vol. 12, no. 11, pp. 2239-2242, 1994.

[86] C. H. Takimoto, "Antifolates in clinical development," Seminars in Oncology, vol. 24, no. 5, supplement 18, pp. S18-S40, 1997.

[87] S. R. Pestieau, O. A. Stuart, and P. H. Sugarbaker, "Multitargeted antifolate (MTA): pharmacokinetics of intraperitoneal administration in a rat model," European Journal of Surgical Oncology, vol. 26, no. 7, pp. 696-700, 2000.

[88] J. Rubin, M. Clawson, A. Planch, and Q. Jones, "Measurements of peritoneal surface area in man and rat," American Journal of the Medical Sciences, vol. 295, no. 5, pp. 453-458, 1988.

[89] A. Chagnac, P. Herskovitz, T. Weinstein et al., "The peritoneal membrane in peritoneal dialysis patients: estimation of its functional surface area by applying stereologic methods to computerized tomography scans," Journal of the American Society of Nephrology, vol. 10, no. 2, pp. 342-346, 1999.

[90] A. M. Albanese, E. F. Albanese, J. H. Miño et al., "Peritoneal surface area: measurements of 40 structures covered by peritoneum: correlation between total peritoneal surface area and the surface calculated by formulas," Surgical and Radiologic Anatomy, vol. 31, no. 5, pp. 369-377, 2009.

[91] C. R. Rossi, M. Deraco, M. De Simone et al., "Hyperthermic intraperitoneal intraoperative chemotherapy after cytoreductive surgery for the treatment of abdominal sarcomatosis: clinical outcome and prognostic factors in 60 consecutive patients," Cancer, vol. 100, no. 9, pp. 1943-1950, 2004.

[92] D. Baratti, S. Kusamura, A. Martinetti et al., "Prognostic value of circulating tumor markers in patients with pseudomyxoma peritonei treated with cytoreductive surgery and hyperthermic intraperitoneal chemotherapy," Annals of Surgical Oncology, vol. 14, no. 8, pp. 2300-2308, 2007.

[93] O. Glehen, V. Schreiber, E. Cotte et al., "Cytoreductive surgery and intraperitoneal chemohyperthermia for peritoneal carcinomatosis arising from gastric cancer," Archives of Surgery, vol. 139, no. 1, pp. 20-26, 2004.

[94] F. N. Gilly, P. Y. Carry, A. C. Sayag et al., "Regional chemotherapy (with mitomycin $\mathrm{C}$ ) and intra-operative hyperthermia for digestive cancers with peritoneal carcinomatosis," Hepato-Gastroenterology, vol. 41, no. 2, pp. 124-129, 1994.

[95] P. Keshaviah, P. F. Emerson, E. F. Vonesh, and J. C. Brandes, "Relationship between body size, fill volume, and mass transfer area coefficient in peritoneal dialysis," Journal of the American Society of Nephrology, vol. 4, no. 10, pp. 1820-1826, 1994.

[96] P. H. Sugarbaker, "Successful management of microscopic residual disease in large bowel cancer," Cancer Chemotherapy and Pharmacology, Supplement, vol. 43, pp. S15-S25, 1999.

[97] J. Esquivel, "Technology of hyperthermic intraperitoneal chemotherapy in the United States, Europe, China, Japan, and Korea," Cancer Journal, vol. 15, no. 3, pp. 249-254, 2009.

[98] S. Van Ruth, V. J. Verwaal, and F. A. N. Zoetmulder, "Pharmacokinetics of intraperitoneal mitomycin C," Surgical Oncology Clinics of North America, vol. 12, no. 3, pp. 771-780, 2003.

[99] W. P. Ceelen, L. Påhlman, and H. Mahteme, "Pharmacodynamic aspects of intraperitoneal cytotoxic therapy," Cancer Treatment and Research, vol. 134, pp. 195-214, 2007.

[100] D. Elias, M. Bonnay, J. M. Puizillou et al., "Heated intraoperative intraperitoneal oxaliplatin after complete resection of peritoneal carcinomatosis: pharmacokinetics and tissue distribution," Annals of Oncology, vol. 13, no. 2, pp. 267-272, 2002.

[101] K. Van der Speeten, O. A. Stuart, H. Mahteme et al., "Pharmacokinetic study of perioperative intravenous ifosfamide," International Journal of Surgical Oncology, vol. 2011, Article ID 185092, 9 pages, 2011. 


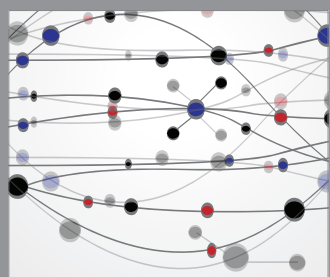

The Scientific World Journal
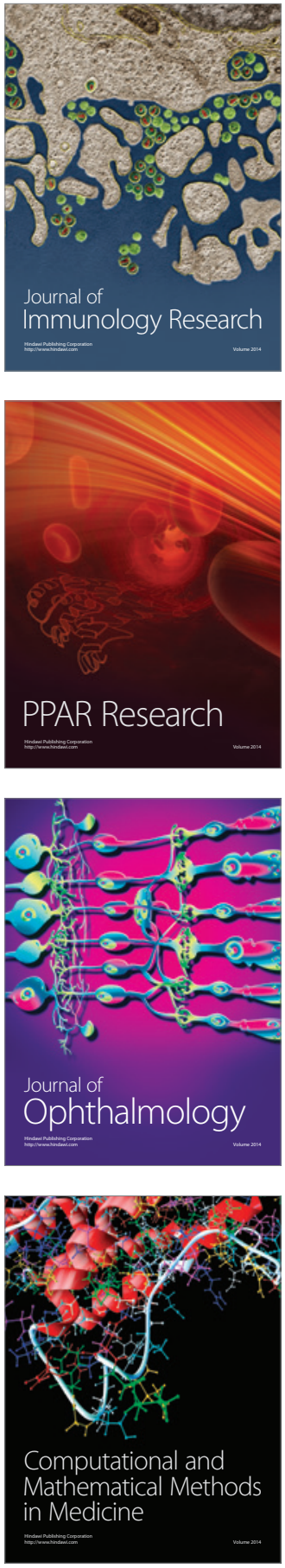

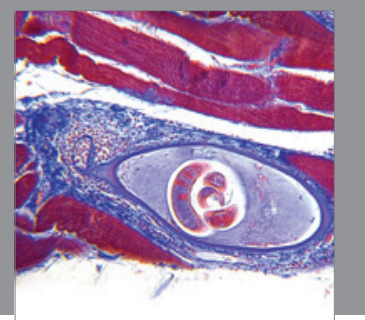

Gastroenterology

Research and Practice
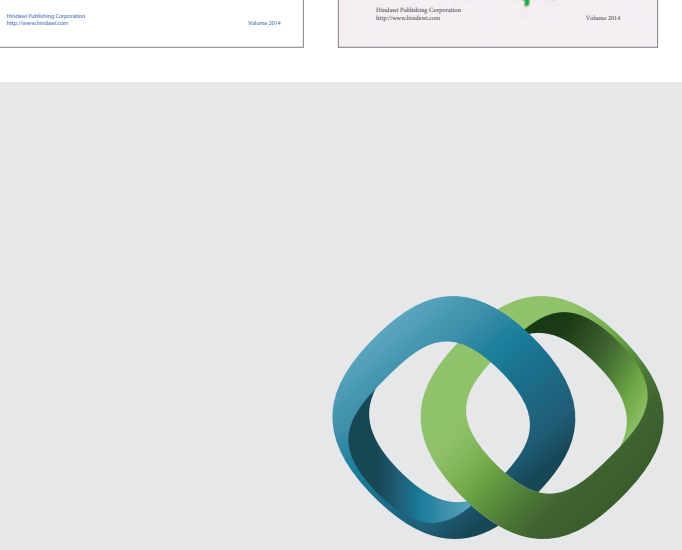

\section{Hindawi}

Submit your manuscripts at

http://www.hindawi.com
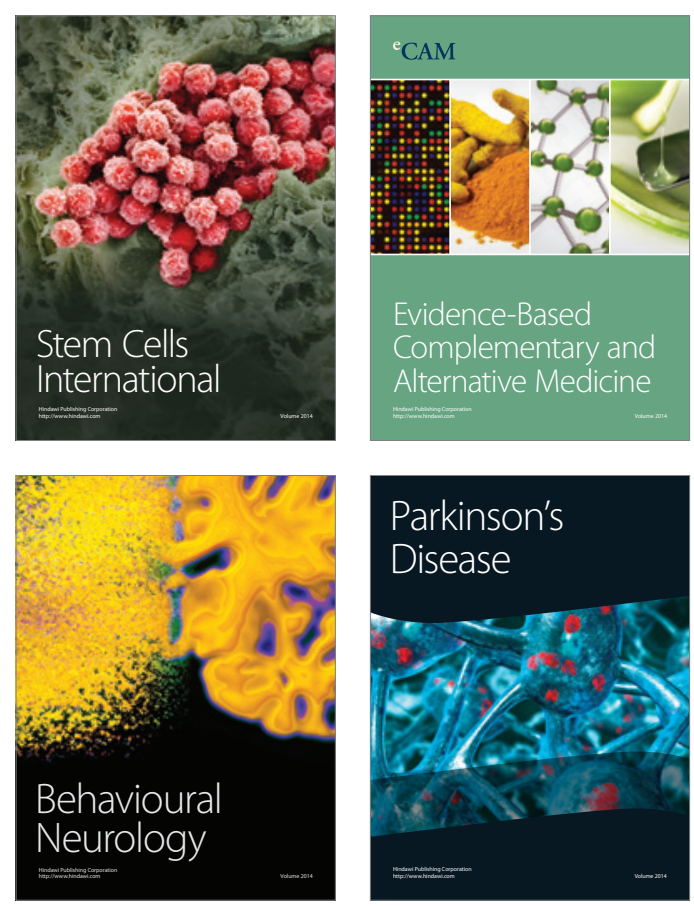

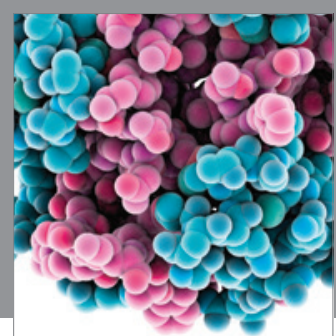

Journal of
Diabetes Research

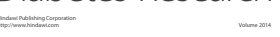

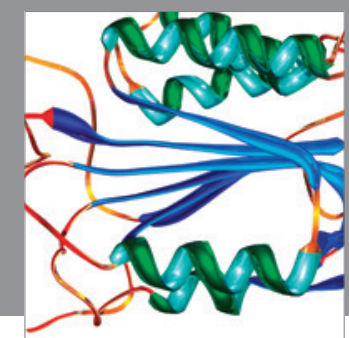

Disease Markers
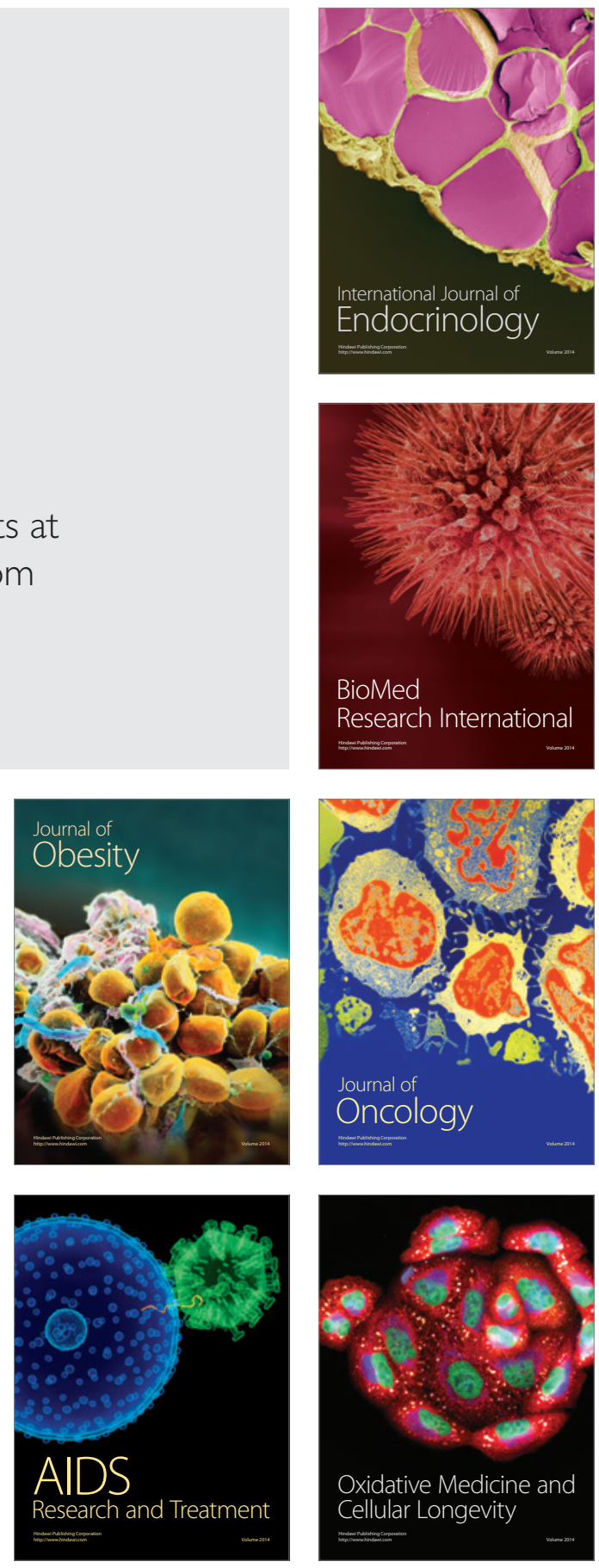